\title{
A picture is worth a thousand words: Electronic cigarette content on Instagram and Pinterest
}

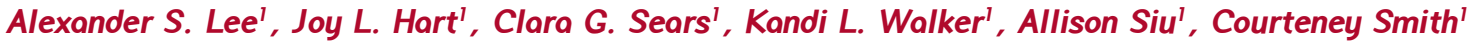

\begin{abstract}
INTRODUCTION This study examined electronic cigarette (e-cig) content in visual materials posted on the social-media platforms Instagram and Pinterest. Both platforms allow users to upload pictures to the internet and share them globally. Users can search for pictures tagged with specific keywords and phrases.

METHODS Using content analysis, this study identified themes in image postings of e-cigs on social media. During five weeks of data collection, keywords were used to identify pictures related to e-cigs. These pictures were then coded into one or more categories.

RESULTS The three most popular categories for Instagram posts were marketing, customization and juices/flavors. The three most popular categories for Pinterest posts were customization, marketing and memes.

conclusions Because of the persuasive power of visuals, it is important to examine communication on Instagram and Pinterest as well as the specific visual messages communicated. Stores and manufacturers use these and similar platforms to communicate with users and potential users; thus it seems that marketers are capitalizing on opportunities for persuasive appeal. The results highlight the popularity of e-cig content on these two social media platforms and reveal an emphasis on marketing and customization.
\end{abstract}

\section{AFFILIATION \\ IUniversity of Louisville, United States \\ CORRESPONDENCE TO \\ Joy L. Hart. University of Louisville, Dept of Communication, Louisville, Ky 40292, United States. E-mail: joy.hart@louisville.edu \\ KEYWORDS \\ social media, e-cigarette Pinterest, Instagram}

\section{INTRODUCTION}

Electronic cigarettes (e-cigs) are handheld battery-operated devices that heat a liquid to generate an aerosol, which is inhaled by the user ${ }^{1,2}$. Over several years, e-cigs have increased dramatically in popularity. For example, in 2015 in the US, 9 million adults vaped and youth used e-cigs more than any other tobacco product ${ }^{3-7}$. The number of disposable e-cigs sold in convenience stores more than quadrupled from 2012 to $2013^{8}$.

Information promoting e-cig brands and products can be easily posted online and shared via social media at virtually no cost to stores or manufacturers ${ }^{9,10}$. Further, consumers can readily share their personal products and experiences. By examining content on Instagram and Pinterest, two popular social media platforms, we can glean a better understanding of e-cig communication.

Instagram is a social media platform that allows users to post photos from smartphones or tablets. These pictures can be tagged with keywords using hashtags. With a keyword search, pictures linked to the hashtags will be displayed, allowing one to explore, comment, or "like" them.

Pinterest is also visually-oriented, and users can "pin" (i.e. post) pictures and save them to their "board" (i.e. typically a collection of related posts, organized by a theme, and tailored to specific interests). People can use hashtags or keywords to search pins and boards created by others, as well as save pins or entire boards. This community-style sharing of pins facilitates the creation and identification of subcultures, hobbies and interests.

The popularity of e-cigs might be driven, in part, by marketing information and personal testimonies widely available to a global audience through these types of social media platforms ${ }^{9-13}$. Although the content of e-cig-related posts on Twitter and Instagram has been analyzed ${ }^{14-18}$, postings on Pinterest rarely have been examined. Analyses of social media posts can increase understanding of e-cig communication, 
which may be helpful in creating and disseminating future health communication campaigns related to e-cig use and potential health dangers.

\section{METHODOLOGY}

\section{Data collection}

Stratified random sampling was used to select images from Instagram and Pinterest over a span of five weeks ( $\mathrm{N}=1800$ pictures). Images were selected from pinterest.com using a computer. Because the Instagram search function is only available on smartphones and tablets, an outside search tool (Statigram, which later became Iconosquare) was used to collect Instagram images via computer.

Twice a week at a random day and time (between 8 a.m. and 11 p.m.), nine different search terms were used to capture a total of 10 pictures from each social media tool, yielding 180 pictures per social media site a week. Originally, ten search terms, accounting for the largest number of posts, were assessed. However, images reached saturation by the ninth search term. Thus, the tenth most popular search term (i.e. electroniccigarettes) was removed because no new images were gathered as a result of this search; retaining it would have led to resampling/recoding of images.

In order to limit the impact of "spam posting" (when retailers flood search results with their advertisements) on the study sample, only 10 pictures were captured from each search term. This method limited overrepresentation of the same image with similar messages repeatedly posted by users. After five weeks a total of 900 pictures ( $\mathrm{n}=100$ for each search term) had been captured for each social media tool.

For both social media sites, the nine most popular search terms related to e-cigs were used. These terms, listed by the number of Instagram hits at the time of writing, are "vape" (8,200,037 hits), "vapelife" (4,945,461 hits), "vapor" (2,353,208 hits), "vapelyfe" (3,847,074 hits), "vapeporn" (4,397,348 hits), “ecig” (1,170,096 hits), “vaping” (2,253,321 hits), "ecigs" (53,466 hits), and "electroniccigarette" (47,577 hits).

\section{Data analysis}

After the images were selected, they were classified into 10 categories. The categories were generated using inductive category development ${ }^{19}$. During this process, researchers reviewed 100 photos and recorded phrases (or words) attributed to the image, as well as first reactions or perceived themes represented by the image. After 100 images were coded, words or phrases were grouped into emergent categories (for both Instagram and Pinterest). The categories were then reviewed and revised, and any disagreements were negotiated.

Once the revised 10 categories were finalized, coding was repeated for the first 100 photos to verify the categories were exhaustive. Then, each of the images identified in data collection for Pinterest and for Instagram was coded into one or more categories. During image coding past the first 100 photos, an eleventh category, not previously identified in the initial phase of categorization, was created. A small sample of images fit only into this novel category, marijuana.

This systemic approach allowed for creation of distinct categories while reducing overlap and redundancy. The categories that resulted from this approach were customization, juice/flavors, memes, marketing, celebrities, health benefits, anti-smoking, models, tricks, marijuana, and social acceptance. The categories are described briefly in the results section and the frequency of images in each category is noted.

\section{RESULTS}

\section{Description of categories}

One of the most popular categories was customization (50.8\% of all images). Users frequently shared information on customizing, or modifying, e-cig devices for both functional and aesthetic purposes.

Numerous images were devoted to marketing e-cigs (53.1\%). Such images were posted by retailers and manufacturers and designed to pique consumer interest in sampling or purchasing particular products.

Another category conveyed information on juice or flavors that can be used in e-cigs (9.72\%). These images described popular and new flavors as well as gave ideas on creating novel flavors.

Some images displayed e-cig use by celebrities (3.11\%). Through such postings, e-cigs are depicted as "hip" and "cool", legitimizing their use.

In a related vein, some postings featured professional or amateur models using e-cigs (8.22\%). Typically these images were seductive or provocative, and involved endorsing products, enticing use, and/or garnering attention.

Some images conveyed perceived health benefits associated with e-cig use $(2.66 \%)$. These posts included personal experiences as well as manufacturer claims.

Further, some images touted e-cigs as a replacement for traditional cigarettes and asserted that e-cig use is better (4.83\%). Beyond potential health benefits, economic and environmental savings were suggested.

A meme is a picture accompanied by text (e.g. catchphrase) that is shareable online and has the potential to go "viral". On both Instagram and Pinterest, memes often served comedic 


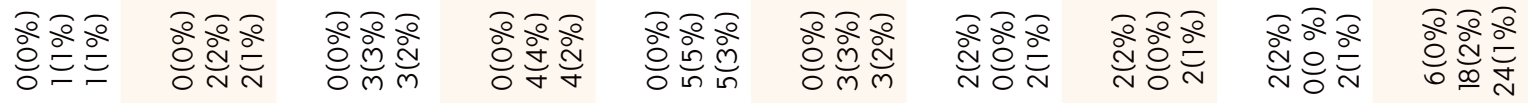

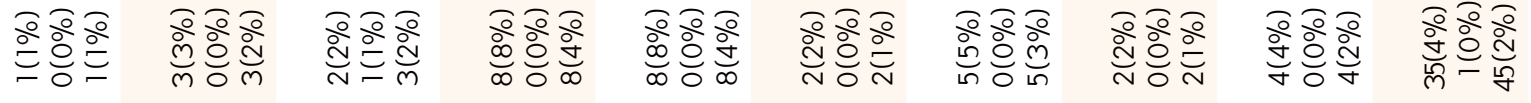

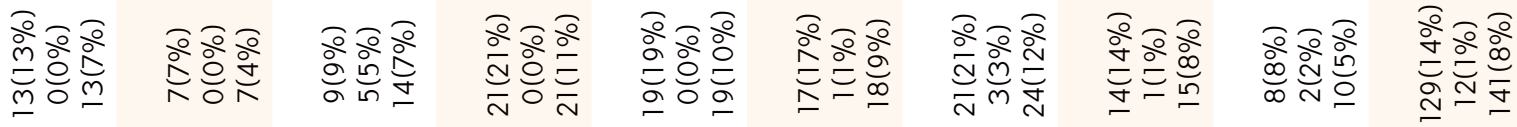

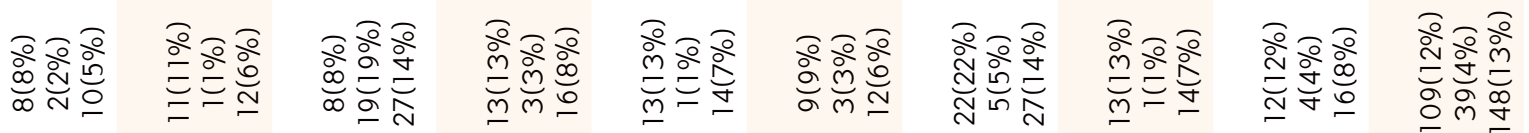

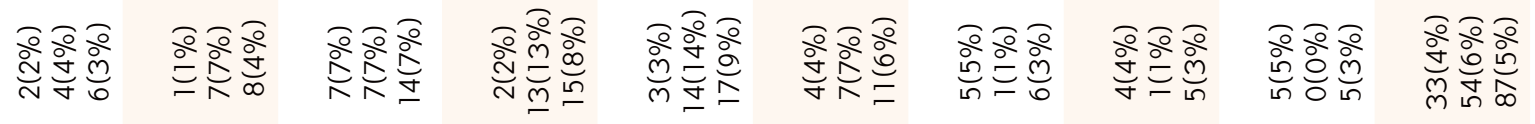

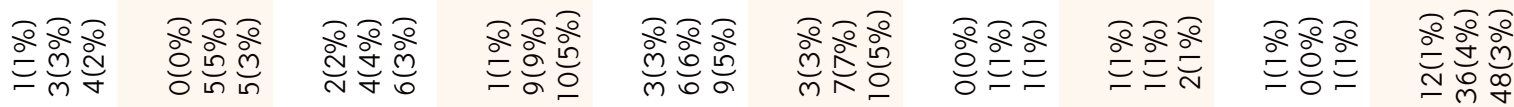

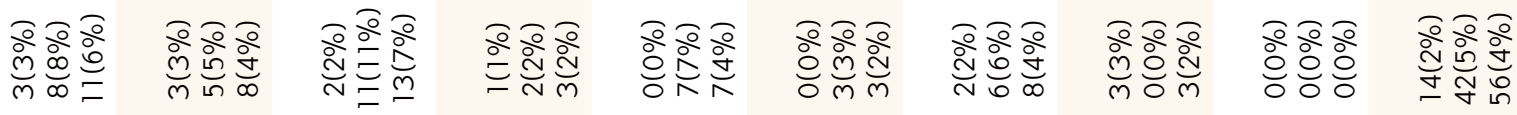

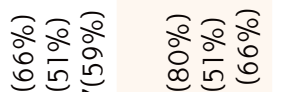

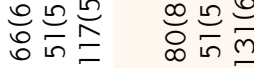

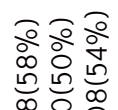
产

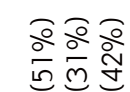

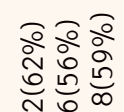

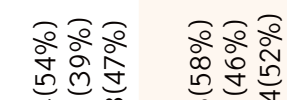

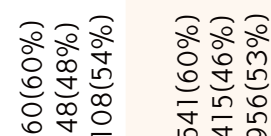

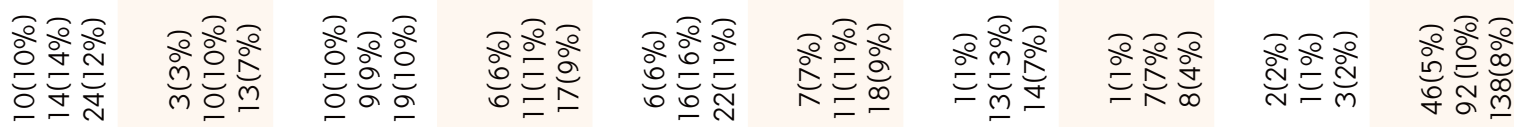

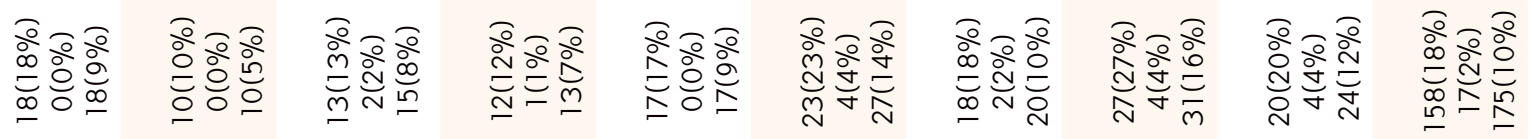

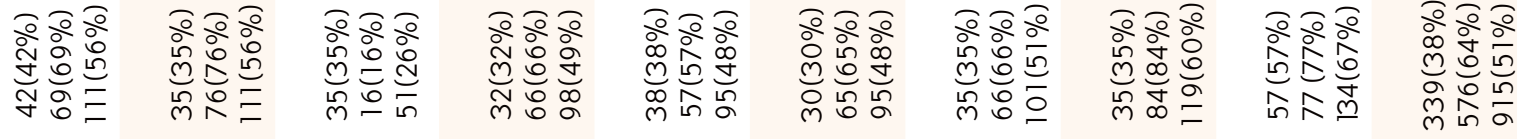

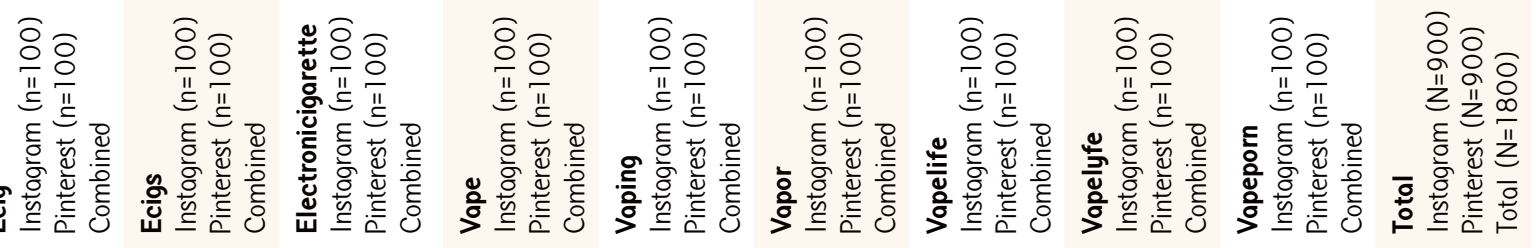


purposes, while simultaneously delivering another message (7.67\%).

Some users uploaded pictures of performing tricks (7.8\%). These tricks consist of exhaling vapor in creative ways, such as producing vapor rings or blowing vapor out the mouth and sucking it back into the nose ("a waterfall").

With cigarette use indoors and in public places often banned, images may be posted to convey social acceptance of e-cigs (e.g. convenient to use in cigarette restricted areas). It is likely that in the hope of attracting potential customers swayed by the social acceptance of e-cigs, companies posted these types of images (1.33\%).

Some images dealt with marijuana use or the potential for such use in e-cig devices $(2.0 \%)$. Often, these images shared updated technology for achieving the best "high" or experiences.

\section{Image frequency by category}

Similar findings emerged for Instagram and Pinterest (see Table 1). For example, the most popular image categories on both platforms were marketing and customization. Results for Instagram indicate the three most popular categories were marketing (60.11\%), customization (37.67\%) and juice/ flavors (17.56\%). On Pinterest, the three most popular were customization (64.00\%), marketing (46.11\%) and memes (10.22\%).

\section{DISCUSSION}

The results highlight the popularity of e-cig content on these two social media platforms, revealing emphasis on marketing and customization. On Instagram and Pinterest, many images (60\% and $46 \%$, respectively) were posted with marketing intentions, indicating broad target audiences and desire to increase sales.

On Pinterest, nearly two-thirds of users (64\%) uploaded photos of customized e-cigs or customizing products. Customization was also popular on Instagram with over onethird of pictures (38\%) focusing on this topic. Given that these social media platforms embrace creativity and art, perhaps it is not surprising that customization was featured.

Particular postings on Instagram may be especially attractive to youth. For example, $18 \%$ of the Instagram pictures related to juices/flavors and 13\% related to performing tricks. Such postings might pique the interest of young people. In particular, traditional cigarette flavors are attractive to youth and increase their willingness to try cigarettes ${ }^{20}$. Although flavored traditional cigarettes are currently banned, e-cig flavors are not and may be attractive to youth. Research has found that advertisements for flavored e-cigs on Twitter were more likely to be re-tweeted than ads for unflavored e-cigs ${ }^{13}$. At present, e-cig advertisements are not restricted in the same fashion as ads for traditional tobacco products, and e-cig advertisements on social media sometimes make claims (e.g. safety, cessation tool) with limited or no justification ${ }^{14}$. In one study, Twitter accounts dedicated to promoting tobacco cessation endorsed e-cigs in $43 \%$ of their tweets ${ }^{15}$. It is possible that interest in flavors, juices, or learning how to perform e-cig tricks would entice youth and others to try e-cigs. In combination with unverified health claims, fear of harmful effects would be minimized or eliminated, thus removing a potential barrier to initiation.

Given the popularity of Instagram and Pinterest, examining e-cig related content on these two platforms increases understanding of e-cig communication; however, our study has several limitations. Firstly, access to some information is restricted for each platform. For example, only the name of the user who posted the photo, the date and time of posting, and any keywords or comments included are available. Often it is not discernible whether the poster is an employee of a shop or a supportive consumer. Key demographics such as age and gender cannot be collected from these social media platforms. Secondly, content on some social media platforms may be analyzed using existing textual analysis programs, but, because Instagram and Pinterest are visually-oriented, we chose manual coding. Thirdly, our approach of selecting terms and sampling shaped the data collection and hence our results.

Because of the subtle persuasive power of visuals, it is important to examine these methods of communicating (i.e. Instagram and Pinterest) as well as the specific visual messages communicated, especially with the popularity of both social media and e-cigs among youth. Understanding e-cig information conveyed on Instagram and Pinterest will better inform researchers, health practitioners and policy makers. Further, this investigation, one of the first to examine e-cig content on Instagram and Pinterest, lays the groundwork for future research. For example, future inquiry could examine messages repeated across multiple social-media platforms, audience interpretations of e-cig messages communicated via social media, as well as the potential use of visual information and infographics to communicate some of the negative health effects of e-cigs as a part of health communication campaigns.

\section{REFERENCES}

1 Noel JK, Rees VW, and Connolly GN: Electronic cigarettes: a new ‘tobacco' industry. Tob Control 2011, 20(1): 81. doi: $10.1136 /$ tc.2010.038562.

2. U.S. Food and Drug Administration. Vaporizers, e-cigarettes, and other electronic nicotine delivery systems (ENDS). Tobacco Products, 
2016. Retrieved from http://www.fda.gov/TobaccoProducts/ Labeling/ProductsIngredientsComponents/ucm456610.htm

3. Centers for Disease Control and Prevention. E-cigarette use triples among middle and high school students in just one year. CDC Newsroom, 2015. Available at: http://www.cdc.gov/media/ releases/2015/p0416-e-cigarette-use.html (accessed June 2017)

4. Centers for Disease Control and Prevention. E-cigarette use more than doubles among U.S. middle and high school students from 2011-2012. CDC Newsroom, 2015. Available at: http://www. cdc.gov/media/releases/2013/p0905-ecigarette-use.html (accessed February 2017)

5. Singh T, Arrazola RA, Corey CG, et al: Tobacco use among middle and high school students — United States, 2011-2015. Morb Mortal Wkly Rep 2016, 65(14): 361-367. doi: 10.15585/mmwr.mm6514a1

6. Regan AK, Promoff G, Dube SR, and Arrazola R: Electronic nicotine delivery systems: adult use and awareness of the 'e-cigarette' in the USA. Tob Control 2011, 22(1): 19-23. doi: 10.1136/tobaccocontrol-2011-050044

7. CDC, "Quick stats: cigarette smoking status among current adult e-cigarette users, by age group-National Health Interview Survey, United States, 2015," Morb Mortal Wkly Rep 2016, 65(42): 1177. doi: 10.15585/mmwr.mm6542a7

8. Loomis BR, Rogers T, King BA, Dench, DL, Gammons DG, Fulmer EB, and Agaku IT: National and state-specific sales and prices for electronic cigarettes-U.S., 2012-2013. Am J Prev Med 2016, 50(1): 18-29. doi: 10.1016/j.amepre.2015.05.003

9. Sears SG, Walker KL, Hart JL, Lee AS, Siu A, and Smith C: Clean, cheap, convenient: promotion of electronic cigarettes on YouTube. Tob Prev Cessation 2017, 3: 10. doi: $10.18332 / \mathrm{tpc} / 69393$

10. Luo C, Zheng X, Zeng DD, and Leischow S: Portrayal of electronic cigarettes on YouTube. BMC Public Health 2014, 14: 1028. doi: 10.1186/1471-2458-14-1028

11. Callahan-Lyon P: Electronic cigarettes: human health effects. Tob Control 2014, 23: 36-40. doi: 10.1136/tobaccocontrol-2013-051470

12. Etter J, Bullen C, Flouris AD, Laugesen M, and Eisenberg T: Electronic nicotine delivery systems: a research agenda. Tob Control 2011, 20(3): 243-248. doi: $10.1136 /$ tc.2010.042168

13. Caponnetto P, Campagna D, Papale G, Russo C, and Polosa R: The emerging phenomenon of electronic cigarettes. Expert Rev of Respir Med 2012, 6: 63-74. doi: 10.1586/ers.11.92

14. Cole-Lewis H, Pugatch J, Sanders A, Varghese A, Posada S, Yun C, Schwarz M, and Augustson E: Social listening: a content analysis of e-cigarette discussions on Twitter. J of Med Internet Res 2015, 17(10): 243. doi: 10.2196/jmir.4969

15. Chu K, Unger JB, Cruz TB, and Soto D: Electronic cigarettes on Twitter - Spreading the appeal of flavors. Tob Regul Sci 2015, 1: 36-41.

doi: $10.18001 /$ TRS.1.1.4
16. Huang J, Kornfield R, Szczypka G, and Emery SL: A cross-sectional examination of marketing of electronic cigarettes on Twitter. Tob Control 2014, 23(Suppl 3): 26-30.

doi: 10.1136/tobaccocontrol-2014-051551.

17. Prochaska JJ, Pechmann C, Kim R, and Leonhardt JM: Twitter=Quitter? an analysis of Twitter quit smoking social networks. Tob Control 2012, 21(4): 447-449. doi: $10.1136 /$ tc.2010.042507

18. Laestadius LI, Wahl MM, and Cho YI: \#Vapelife: An exploratory study of electronic cigarette use and promotion on Instagram. Subst Use Misuse 2016, 51(12): 1669-1673. doi: 10.1080/10826084.2016.1188958

19. Mayring P: Qualitative content analysis. Forum: Qualitative Social Research 2000, 1. Available at: http://www.qualitative-research. net/index.php/fqs/article/view/1089 (accessed February 2017)

20. U.S. Food and Drug Administration. Candy and fruit flavored cigarettes now illegal in United States; Step is first under new tobacco law. News \& Events, 2009. Available at: http://www.fda. gov/NewsEvents/Newsroom/PressAnnouncements/ucm183211. htm (accessed February 2017)
CONFLICT OF INTERESTS

The authors have

completed and submitted the ICMJE Form for Disclosure of Potential Conflicts of Interest and none were reported.

\section{FUNDING}

This work was supported in part, by the National Heart, Lung, and Blood Institute (NHLBI) and FDA Center for Tobacco Products (CTP) (grant number 5P5OHL120163).

The project also received support from the Department of Communication at the University of Louisville. FDA funding pertained only to aspects of the project that were under the CTPFDA regulatory authority at the time of the dato collection. The content is solely the responsibility of the authors and does not necessarily represent the official views of the $\mathrm{NIH}$ or the Food and Drug Administration

PROVENANCE AND PEER REVIEW

Not commissioned; externally peer reviewed 